ON THE

\title{
USE OF ARSENIC
}

IN SOME

\section{AFFECTIONS OF THE UTERUS.}

\section{BY HENRY HUNT EsQ.,}

OF DARTMOUTH.

Communicated by Mr. PERRy.

READ MARCH 27TH, 1838.

In 1834, the following case, whose result first attracted my attention to the effects of arsenic on the uterus, occurred to me.

A woman, about forty years old, consulted me for a cancer of the uterus in a state of ulceration, accompanied by a profuse fetid discharge, and great suffering, from which she was rapidly sinking. I prescribed for her four drops of the liquor arsenicalis three times a day, gradually increasing the dose to ten drops, which she continued a fortnight, when the well known poisonous effects of that mineral presented themselves, and in the same proportion the pain in the womb subsided. The discharge, however, continued unabated; she recovered slowly from the effects of the arsenic, but up to the time of her leaving Dartmouth, which was nearly two months, she had no return of pain in the uterus. I never could ascertain how the case terminated, but conclude that it ended fatally. 
The fact of the pain in the uterus of this poor woman being relieved, as soon as the arsenic affected her system, struck me forcibly, and recollecting that inflammation of the genitals occasionally follows its administration as a poison, I was induced to hope it might be useful in some disorders of those parts. I was further encouraged by Dr. Locock, to whom I related the preceding case, who told me that he had cured a lady of menorrhagia by arsenic, having recommended it to her for a disorder of the nose, being ignorant at the time that she was subject to the former disorder; she having neglected to mention it, considering it quite irremediable, as it had baffled the skill of every physician she had hitherto consulted.

The following cases, the results of my experiments, will shew that my hopes were not altogether unfounded.

Mrs. N., ætat. 42, was subject when young, to hysteria, but was otherwise strong and healthy. She continued well up to the time of her third pregnancy, about sixteen years ago, when she became so enormously distended, as to cause considerable anxiety, her size being afterwards accounted for by her being delivered of three children. Her recovery was slow; for many months she was unable to sit upright, and has never since regained her former strength, having been kept in a state of great debility by profuse menstruation. The discharge was not only too profuse, but continued eight or ten days uninterruptedly, and returned again at the end of a fortnight, or sooner, if she had any anxiety or 
fatigue; and her debility was further increased by an irritable state of the bowels, which almost invariably came on three or four days previous to the menstrual period. Many methods of treatment had been tried by various medical men with little benefit, and that little only temporary. In the summer of 1835 , I first gave her liq. arsenicalis ( $\mathrm{Ph}$. Lond.), in doses of four drops three times a day, with ten of the Tinct. Camphoræ comp., commencing immediately after being unwell. The result was most satisfactory, for she did not menstruate again until two days beyond the natural time, and when she did, the discharge continued only five days, and was not more in quantity than it ought to have been; neither did the diarrhœa supervene. She then left off taking the arsenic regularly; but for the three or four succeeding months, she took it again for a week previous to the menstrual period, since which she has continued regular, is stronger and less hysterical than she has been since her confinement.

Mrs. B. P., ætat. 34; married ; of a delicate habit ; has borne. seven children, and has miscarried three times. Since the last confinement, which happened two years ago, she has menstruated too frequently and too profusely, and has been also weakened by leucorrhœa, having seldom, during the last year, passed three days together free from the menstrual discharge. Her bowels have also been much relaxed, her tongue red and shining, and she has been harassed by a troublesome cough, with much expecto. ration : her strength and flesh were so much reduced 
as to make her case a very unfavourable one. I gave her a pill containing the twentieth of a grain of the arsenious acid, with a draught, containing Liq. Calcis 3ss. Syrup. Sarsæ 3iij. three times a day. She commenced taking the medicine on the 27th of September, 1837, and for three weeks there was no improvement, excepting that the bowels were less relaxed. On the 19th of October, the menstrual discharge ceased, and did not return until the 28th of November, when she menstruated naturally and for six days only. She continued taking the medicine one month longer, and has now regained her usual strength and flesh, her cough has almost ceased, and she has since menstruated at the proper time. This lady had previously consulted three or four medical men, without deriving benefit from their prescriptions.

Mrs. W. H., ætat. 42, suffered in her youth for many years from dysmenorrhœa and hysteria, but was otherwise well and strong, until about six years ago, when she was much reduced by a severe and obstinate diarrhœa, which yielded at length to the remedies administered, but not until her strength was so much exhausted that she could with difficulty walk across her room. This lady expresses herself so clearly, that I will give in her own words that part of her case which more particularly relates to the subject of this paper. After having given a description of her illness above referred to, she says, "It was certainly two years or more before I became irregular; I then began to be unwell every fortnight, 
IN SOME AFFECTIONS OF THE UTERUS. 281

and continued so, violently, a whole fortnight. It was after this that I began to take the pills, (containing the same quantity of arsenic as those given in the last case,) which seemed at once to increase my appetite and give me a kind of energy, that made it much less irksome to move. When I had taken the pills about three months, my strength was much increased, and from the time that I began taking them, I gradually became more regularly unwell. I left off the pills some months and then began them again, in consequence of a return of weakness and of being again too often unwell, and they were certainly of great benefit to me." This lady has since continued well and is now capable of riding eight or ten miles on horseback, without much fatigue, and menstruates regularly.

Eliz. Stubbs, ætat. 17, menstruated, for the first time, at the age of 15 , for a few months regularly, since which the discharge has returned too often, and for the last year it has continued almost constantly, and in such profusion as to debilitate her greatly, and to oblige her to leave her situation as a servant, although healthy in every other respect. I was consulted in August, 1837, and gave her the arsenic in pills, as in the two former cases, one three times a day. The discharge was arrested after she had taken the pills a fortnight, and returned at the proper time; since which she has continued to menstruate regularly, and has regained her strength. She took the pills six weeks. 
Eliz. Locke, ætat. 36, unmarried, was strong and healthy until two years ago, when she was much exhausted by fatigue and loss of rest. From that time her strength has been gradually declining, which she attributes to the circumstance of her being unwell profusely for six or eight days at a time, with only a week intervening between the periods :-in other respects she is well. On the 16th November she commenced the arsenical pills, taking one three times a day. The discharge ceased two days afterwards, too early to be attributed to the effect of the medicine ; after three weeks it returned, not, however, so copiously as before, and continued only five days. She has since only menstruated once, at the proper time, and has resumed her situation as a servant.

Eliz. Fox, ætat. 17, consulted my father in 1836, for a leprous eruption on the knees and elbows, for which he prescribed three drops of the liquor arsenicalis three times a day. At the end of three months her mother called on him, to thank him for not only curing her daughter of the eruption, but for making her regular, then mentioning, for the first time, that previously to taking the medicine, her daughter had menstruated much too frequently and profusely, which had gradually abated since she had commenced taking the drops; and that she was then quite well.

It is almost unnecessary to state that there was no inflammation or organic disease of the uterus of those females whose cases have been related, but the excessive flow of the uterine discharge appeared to be the 
IN SOME AFFECTIONS OF THE UTERUS. 283

consequence of exhaustion, (except in the two younger girls,) although the exhaustion originated in the several individuals from different causes.

From the immediate and progressive improvement which succeeded the administration of the arsenic, the cessation of the menorrhagia may be fairly attributed to the action of that medicine, and its peculiar power is more clearly shewn in the first three cases, for in two, the disorder had previously resisted every remedy that had been given under the direction of very skilful physicians; and in the third, the immoderate discharge was arrested while taking the arsenic the first time, but returned soon after it had been left off, and was again immediately and permanently checked by resuming it.

The success attending the use of this remedy in menorrhagia induced me to give it in some other affections of the uterus, some examples of which are here annexed.

Mrs. Burne, ætat. 30, married, but never pregnant, was labouring under the following symptoms when she consulted, me in June, 183\%. A constant pain with heat, varying in severity, in the lower part of the sacrum, in the left groin, and under the pubes, and bearing down, which was much increased by walking, standing or sitting upright, by a costive state of bowels, or by the action of purgative medicine. She was easier in bed or reclining on a sofa; her urine was sometimes high coloured, at other times pale, her pulse rather quickened. On examination, per vaginam, the uterus was found to be 
tender and rather tumid. She attributed her disorder to the menses having been suddenly checked by exposure to cold three years before. She had consulted many medical men, who had bled her generally and locally, and always with temporary relief; purgatives, opiates, the warm and cold baths, and numerous other remedies, having been employed without any lasting benefit.

Thinking this to be a case of chronic inflammation of the uterus, I confined the patient to her bed, and for six weeks kept her under the influence of a mild course of mercury with nitre and colchicum. While she remained in bed she was easy, but the pain returned with equal severity immediately on her leaving it and taking exercise. I then sent her home, and directed a pill, with $\frac{1}{20}$ of a grain of arsenic, to be taken three times a day, which she continued four months, at the end of which time the pain, which had gradually decreased after she had taken the pills six weeks, entirely ceased. She now attends to the active duties of a bakehouse, and only suffers pain about the time of her menstruation. This case may perhaps be considered similar to those described by Dr. Gooch as the irritable uterus.

Mrs. W., ætat. 44, married, a strong and robust lady, has borne five children, the birth of the last, about six years ago, was followed by hæmorrhage so profuse as to endanger life, to arrest which it was necessary to introduce the hand into the uterus, to remove the coagula, and to procure its contraction. This was followed by inflammation of the uterus, 
which was with difficulty subdued; she was, when convalescent, necessarily much reduced in strength, and her recovery procrastinated by leucorrhœe. After a few months she menstruated, but for three or four days before the discharge came on she suffered acutely from neuralgic pain in the face. This regularly returned a few days previously to her being unwell, each time increasing in severity, until it became most agonizing, though in the intervals she was quite easy. To relieve this pain, I gave her five drops of the liquor arsenicalis three times a day, gradually increasing the dose as largely as it could be borne. She took it for three months with an occasional interval of a few days. The violence of the paroxysm was mitigated, but still it returned regularly for several months longer, although each attack became less acute than the preceding one. The medicine having become very repugnant to her, was discontinued for a few weeks, and only given in small doses for four or five days every month previously to her being unwell. This plan was pursued for several months after the pain had ceased, in consequence of her feeling a peculiar sensation in the cheek, which always preceded the paroxysm. During the period of her suffering so severely, there was no apparent deviation from health in the menstrual discharge. She remains well.

Although in this case there was no apparent disorder of the uterus to cause the attack of neuralgia, yet, when it is remembered that this organ suffered so 
severely at and after delivery, that the pain came on regularly at the menstrual period, and that it was quite absent in the intermediate time, it may be fairly inferred that some irritation in the uterus was the cause of it, and further, that the arsenic removed it by overcoming that irritation. At the time when this lady was under my care, I was ignorant of the effect of arsenic on the uterus, but since my attention has been directed to it, I have relieved several ladies suffering in a similar manner, whose cases should be detailed, were it not that this paper would be extended to too great a length.

Dr. F. H. Ramsbotham, in his excellent Lectures on Midwifery, states that "It is occasionally observed, that the menses appear in particular persons very irregularly, sometimes occurring at the end of a fortnight, at others, not for five or six weeks; they are also irregular in regard to the time they continue flowing, and to the quantity produced at each period." This he considers only inconvenient, and that no means should be taken to regulate the return. The following case is one of that description, with the exception of the lady's having suffered much pain and general disorder when the menses did not return at the proper period.

Mrs. H., ætat. 34, was for many years irregular; the menses seldom returned too frequently, but generally at the end of five or six weeks, or even longer. When this happened, she had much pain in the loins, head, or chest, or under the sternum, but never simulta- 
IN SOME AFFECTIONS OF THE UTERUS. 287

neously; sometimes it attacked one part, then suddenly quitting it, it attacked another, varying frequently during the day.

These erratic pains were more severe, the longer the return of the menses was postponed, but the instant they came on she was quite relieved. The menses seldom continued to flow regularly, but sometimes suddenly stopped, when the pain in one part or the other immediately returned. She took three drops of the liquor arsenicalis twice a day for three months in the summer of 1837 , and at each succeeding period she suffered less, and the discharge has since returned quite regularly.

By examining the effect of arsenic when given in poisonous doses, its beneficial action in the foregoing cases may be somewhat explained, for it appears, according to the observation of Professor Christison, that the genital organs are affected if life is prolonged beyond three days after taking arsenic as a poison, in which case irritation in the other mucous passages succeeds or accompanies the signs of, inflammation of the alimentary canal; and in his work on poisons, he states, at page 217, "In many instances, too, the urinary passages are affected, the patient being harassed with frequent, painful, and difficult micturition, swelling of the penis, and pain in the region of the bladder; or if in a female, with burning pain in the vagina and excoriation of the labia, but this does not happen unless life is prolonged beyond three days." At page 240, in a case related by Bachman, "the woman complained of burning of the fundament and 
genitals, both of which were inflamed even to gangrene;" again at page 244, "In ordinary cases in which death is delayed till the second day or later, a considerable variety of diseased appearances has been observed; they are different changes of structure arising from inflammation of the alimentary canal, in the organs of the chest, and the organs of generation." At page 253, "the organs of generation are occasionally affected; in a case related in Pyl's collection, the inside of the uterus and fallopian tubes were inflamed."

Although inflammation of the inside of the uterus has been only observed in one case, yet it may be reasonably inferred that in cases mentioned by Professor Christison, where inflammation in the genitals had been so violent as to terminate in gangrene, inflammation of the uterus would have been observed, if that organ had been examined.

If the foregoing observations are correct, the benefit derived from this medicine may be explained by its acting on the mucous membrane of the uterus as a stimulant. It follows that the cases in which it would be given with the greatest advantage, are those in whom the disordered condition of the uterus had been induced and kept up by debilitating causes.

As it is frequently desirable to continue the use of this remedy for a considerable time, for large doses taken for a short time produce much distress without the desired effect on the uterus, the form in which it is most easily borne by the stomach should be selected, and I have observed that when it is given in pills 
containing $\frac{1}{20}$ of a grain, it has produced less inconvenience than the common preparation, the liquor arsenicalis; I have therefore generally chosen that mode of giving it, and my patients have seldom complained of any unpleasant feeling from it, although in some instances it has been taken for many months in succession.

The stomach however does not become accustomed to it, as it does to many other remedies, so as to bear a gradual and continual augmentation of the doses; but on the contrary, the longer it is continued the greater is the inconvenience it occasions, so that instead of increasing, it is often necessary to lessen the quantity, after it has been taken some little time, or even to discontinue it for a week or two, and then resume it. Some individuals are much more sensible to its effects than others, but the most sensitive, by taking the pill immediately after meals, have been enabled to continue it as long as it has been necessary, while others can take two pills, or $\frac{1}{10}$ of a grain, three times a day for a considerable period, without any unpleasant effect.

I need scarcely remark that great care is necessary in making the pills, in order that the arsenic may be evenly divided.

I have often combined it with other remedies with manifest advantage, but I have selected the foregoing cases as they were cured by arsenic alone. 